\title{
A pilot study of ultrasound-guided electronic brachytherapy for skin cancer
}

\author{
Uma Goyal, MD!, Yongbok Kim, PhD!', Hina Arif Tiwari, MD², Russell Witte, PhD², Baldassarre Stea, MD, PhD' \\ 'Department of Radiation Oncology, ${ }^{2}$ Department of Radiology, University of Arizona, Tucson, Arizona, USA
}

\begin{abstract}
Purpose: Electronic brachytherapy (eBT) has gained acceptance over the past 5 years for the treatment of non-melanomatous skin cancer (NMSC). Although the prescription depth and radial margins can be chosen using clinical judgment based on visual and biopsy-derived information, we sought a more objective modality of measurement for eBT planning by using ultrasound (US) to measure superficial (<5 mm depth) lesions.

Material and methods: From December 2013 to April 2015, 19 patients with 23 pathologically proven NMSCs underwent a clinical examination and US evaluation of the lesions prior to initiating a course of eBT. Twenty lesions were basal cell carcinoma and 3 lesions were squamous cell carcinoma. The most common location was the nose (10 lesions). A 14 or $18 \mathrm{MHz}$ US unit was used by an experienced radiologist to determine depth and lateral extension of lesions. The US-measured depth was then used to define prescription depth for eBT planning without an added margin. A margin of $7 \mathrm{~mm}$ was added radially to the US lateral extent measurements, and an appropriate cone applicator size was chosen to cover the target volume.

Results: The mean depth of the lesions was $2.1 \mathrm{~mm}$ with a range of 1-3.4 $\mathrm{mm}$, and the mean largest diameter of the lesions was $8 \mathrm{~mm}$ with a range of 2.6-20 mm. Dose ranged from 32-50 Gy in 8-20 fractions with a median dose of 40 Gy in 10 fractions. All patients had a complete response and no failures have occurred with a median follow-up of 12 months (range of 6-22 months). Also, no prolonged skin toxicities have occurred.

Conclusions: A routinely available radiological US unit can objectively determine depth and lateral extension of NMSC lesions for more accurate eBT treatment planning, and should be considered in future eBT treatment guidelines.

J Contemp Brachytherapy 2015; 7, 5: 374-380

DOI: 10.5114/jcb.2015.55538
\end{abstract}

Key words: electronic brachytherapy, skin cancer, ultrasound.

\section{Purpose}

Non-melanomatous skin cancer (NMSC) affects over 3.5 million patients per year in the United States [1]. Treatment of NMSC can have significant effects on quality of life and cosmesis. There are several available treatment options including surgery, topical chemotherapies, external beam radiation therapy, and brachytherapy $[2,3,4$, $5,6]$. Although effective, surgery can be disfiguring and morbid for lesions located on cosmetically sensitive areas of the body such as the face [2]. For these lesions and for medically inoperable patients, radiotherapy can offer an equally effective alternative to surgery.

Several radiation techniques are available to treat NMSCs. When using conventional megavoltage radiation, treatments can last between 3-6 weeks depending on size and location of the lesion [6, 7]. In addition, to ensure adequate margins and dose to the skin surface, water-equivalent bolus and clinical evaluation are commonly utilized $[6,7]$. Superficial/orthovoltage radiation $(40-300 \mathrm{kVp})$ has also been used to treat thin superficial NMSC $[8,9]$.
Finally, for large tumors with irregular shapes, highdose-rate (HDR) brachytherapy molds, flaps, interstitial catheters, or radionuclide applicators can be used with an ${ }^{192}$ Ir source [3, 5, 10, 11, 12, 13, 14, 15].

Since 2009, electronic brachytherapy (eBT), a $50 \mathrm{kVp}$ miniaturized $X$-ray source with specialized cone-shaped applicators has been used to treat superficial NMSC. Xoft eBT (Axxent eBx; Xoft - a subsidiary of iCAD, Inc., Sunnyvale, $\mathrm{CA}$, USA) does not require a radioactive isotope and involves minimal shielding $[16,17,18]$. It also does not require the same regulations of a radioactive source such as ${ }^{192}$ Ir [19]. Xoft eBT uses a hypofractionated regimen as do other orthovoltage or HDR brachytherapy techniques [13, $17,18,20]$. Another system similar to Xoft eBT is Esteya (Elekta AB, Stockholm, Sweden) but uses a $69.5 \mathrm{kV}$ X-ray source $[21,22,23]$.

Because of eBT low energy $X$-rays, the maximum dose is at the surface, and the dose at $5 \mathrm{~mm}$ depth is $50-60 \%$ of the surface dose depending upon the applicator, e.g., if the prescription depth is $5 \mathrm{~mm}$ then $\sim 200 \%$ of dose is at the skin surface $[24,25]$. In a previously published study

Address for correspondence: Uma Goyal, MD, Department of Radiation Oncology, University of Arizona, Received: 05.09.2015 1501 N. Campbell Avenue, Tucson, Arizona, USA 85721, USA, phone: +1 520-694-7427, 
using Xoft eBT for lesions 1-7 $\mathrm{mm}$ thick treated with $40 \mathrm{~Gy}$ in 8 fractions, treatment resulted in a local control of $100 \%$ and an excellent cosmesis in $93 \%$ of patients with no toxicity at a follow-up of 1 year [18]. In this particular study, CT scans were used to estimate the depth of treatment. In many clinics, however, a default depth is selected for eBT calculations and is used universally for all patients. The lateral extension is usually determined with visual inspection on the skin surface and a varying margin is applied based on the physician's discretion [6, 7]. In our initial eBT experience, using clinical estimates of depth and lateral extent, led to complications such as grade 4 skin necrosis and prolonged wound healing, especially with thicker lesions and lower extremity lesions (Figure 1). This motivated us to investigate a more accurate approach in choosing an applicator size and dose depth prescription by utilizing clinically available ultrasound (US) to measure the depth and lateral extent in order to prevent overdosing the skin. Such US methods have been previously investigated with HDR-BT [13].

In the past, NMSC lateral extension and depth measurements have commonly been determined by visual estimation during clinical examination $[6,7]$ but this method can be quite subjective and physician-dependent. Measurements from punch biopsies have also been utilized but have led to questions regarding its accuracy [26].

This study reports on the feasibility of using standard US technology as a measuring tool to determine the diameter and the depth of a lesion in order to adequately prescribe radiation dose in $\mathrm{eBT}$ treatment planning.

\section{Material and methods}

From December 2013 to April 2015, 24 patients with 28 biopsy confirmed NMSCs were referred for definitive Xoft eBT. The depth and lateral extension for all lesions were assessed with US imaging. Five lesions, 4 basal cell carcinomas (BCC), and 1 squamous cell carcinoma (SCC), could not be visualized on US and therefore they were not included in this study. These were treated at a default depth of $1 \mathrm{~mm}$ and with a radial margin based on the biopsy scar.

Due to limitation of the eBT technique (low energy and high surface dose), the lesions treated were limited to a depth of $<5 \mathrm{~mm}$. In addition, the largest available Xoft applicator size is $50 \mathrm{~mm}$ in diameter, so the largest lateral dimension of any treated lesion was limited to 36 $\mathrm{mm}$ due to inclusion of a $7 \mathrm{~mm}$ radial margin to cover microscopic disease and setup errors. Lesions were ineligible if evidence of clinical or pathologic positive lymph nodes, metastatic disease, or melanoma histology.

Radiation oncology and radiology records, referring physician documentation, and hospital records were examined. Data was gathered on: patient demographics, pathology, physical examination, photographs, threedimensional (3D) tumor measurements with US, and radiation treatment planning sheets, calculations, and quality assurance (QA). There were 23 eligible skin lesions in 19 patients for this study. Lesions were staged based on the American Joint Committee on Cancer staging manual $7^{\text {th }}$ edition [27]. Toxic effects were graded according to the National Cancer Institute Common Toxicity Criteria for Adverse Events, version 4 . This retrospective study was approved by our institutional review board. Written consent from each patient was received to publish their data and photographs.

\section{Lateral extension and depth measurements}

A patient with a suspicious skin lesion first underwent a shave biopsy by a dermatologist. After a diagnosis of NMSC was rendered, the patient was referred for radiotherapy and underwent a consultation with a board-certified radiation oncologist. The patient was then sent for US imaging of the lesion for lateral extent and depth measurements, which were documented in a report within an accessible electronic medical record.
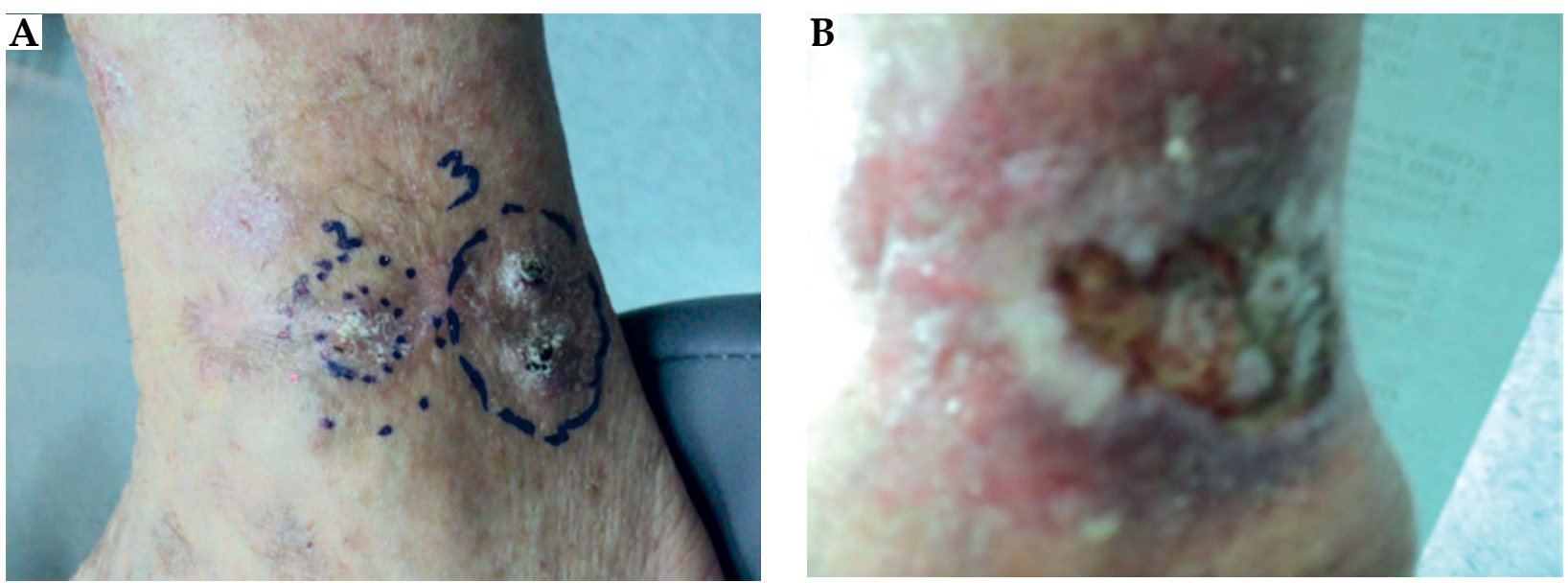

Fig. 1. Default prescription depth toxicity. Two lesions on the lower extremity were treated to $40 \mathrm{~Gy}$ in 8 fractions (5 Gy per fraction) twice per week using a $35 \mathrm{~mm}$ cone based on clinical exam and default depth of $3 \mathrm{~mm}$ in our early electronic brachytherapy (eBT) experiences. The patient developed a non-healing grade 4 ulceration that persisted even after 4 months post-eBT. Although this toxicity could be due to a number of contributing factors, it illustrates the need for a more precise and accurate depth of prescription. A) Pre-treatment, B) 4 months post-eBT 
Focused US of the lesions was performed by a boardcertified radiologist using either: the LOGIQ E9 (GE Healthcare, Milwaukee, WI, USA) or S-3000 US units (Acuson; Siemens Medical Solutions, Mountain View, CA, USA). Linear array transducers with upper frequencies of 14 and $18 \mathrm{MHz}$ were utilized to obtain sonograms. Imaging was performed using either a custom made gel standoff pad or copious amounts of cold US gel acting as a standoff, especially on body parts where the gel pad could potentially cause image degradation due to poor apposition. Scanning was done gently to avoid distortion of lesions. Two-dimensional (2D) B-mode images were acquired with transverse and longitudinal sweeps across the lesion. The US probe was oriented along the longest dimension of the lesion to obtain sagittal and transverse measurements. Measurements of depth were made at the most vertical extension of the NMSC in either plane.

\section{Margin for clinical target volume and planning target volume}

In order to cover potential subclinical disease, a margin of $5 \mathrm{~mm}$ was added radially to the gross tumor volume (GTV) diameter measurements to create the clinical target volume (CTV), even though dermato-pathologic data has shown that the lateral extent estimate derived from US measurements tends to overestimate the extent of the lesion [28]. Since eBT is a contact therapy with the applicator applied directly and firmly to the skin, there was no expected movement during treatment but an inter-fractional error in positioning the applicator was possible. Therefore, a $2 \mathrm{~mm}$ margin was added to the CTV to create a planning target volume (PTV). However, when treating difficult anatomical sites (e.g. eyelid, lip), CTV and PTV were reduced based on practicality and clinical judgment. With 4 eBT applicator sizes available (10, 20, 35 , and $50 \mathrm{~mm}$ ), we used the cone size that adequately covered the PTV.

No extra margin was added to depth based on US measurements and Mohs pathology data showing that US may overcompensate for depth by about $27 \%$ [28], and there was no expected setup error in the vertical dimension.

\section{Radiation doses and fractionation}

The median dose used was 40 Gy in 10 fractions delivered every other day; median fraction size was $4 \mathrm{~Gy}$. Two lesions on the upper lip and tip of the nose received a more protracted course of $50 \mathrm{~Gy}$ in 20 fractions of $2.5 \mathrm{~Gy}$ per fraction due to central anatomical locations and greater depths. One patient stopped her treatment after $32 \mathrm{~Gy}$ due to grade 3 erythema.

\section{Treatment procedure}

During simulation, an immobilization device was fabricated depending upon the location of the NMSC. Patients with lesions on the face were immobilized using an aquaplast facemask (CIVCO Medical Solutions, Coralville, IA, USA) and the area to be treated was cut out from the facemask. Lesions on the extremities were stabilized using a knee roll or wedge cushion. Photographs were taken at the time of setup to aid in treatment setup verification.

When using eBT, the maximum dose occurs at the skin surface due to the use of low energy X-rays, therefore output of the source was defined as the dose rate (Gy/minute) at the skin surface for each applicator. This X-ray source calibration for each applicator followed the American Association of Physicists in Medicine (AAPM) task group (TG) 61 protocol [24, 25]. The beam-on time was computed by the ratio of prescribed dose over the product of dose rate and vendor provided percentdepth-dose (PDD) values. Therefore, for a given prescription dose, 2 parameters were essential in treatment planning calculations: prescription depth determined the PDD value while the lateral extension determined the applicator size.

At the time of treatment, after the patient was setup on the treatment couch, the lesion was demarcated with a fine tip marker by the physician and compared to photographs taken during simulation. Then the applicator was placed firmly against the skin and treatment initiated. The patient was monitored by 2 therapists and a physicist who remained in the room to ensure the patient kept the same position as planned. Aloe vera gel (RadiaGel Hydrogel, Medline Industries, Inc., Mundelein, IL, USA) was provided for the treated area during the eBT course and as long as the radiation-induced erythema persisted (usually a month).

\section{Effects on surface dose}

The percent of prescription dose at the surface is considered for clinical purposes (Table 1). Surface dose increases with increasing depth. Cone applicator size with a constant depth also shows small variation in percent of prescription dose at the surface.

Table 1. Skin surface dose

\begin{tabular}{|c|c|c|c|c|}
\hline Prescription depth (mm) & $10 \mathrm{~mm}$ cone & $20 \mathrm{~mm}$ cone & $35 \mathrm{~mm}$ cone & $50 \mathrm{~mm}$ cone \\
\hline 1 & $113.0 \%$ & $115.2 \%$ & $112.1 \%$ & $111.7 \%$ \\
\hline 2 & $127.9 \%$ & $132.5 \%$ & $125.6 \%$ & $124.4 \%$ \\
\hline 3 & $144.5 \%$ & $151.5 \%$ & $140.4 \%$ & $137.9 \%$ \\
\hline 4 & $163.4 \%$ & $172.1 \%$ & $156.7 \%$ & $151.7 \%$ \\
\hline 5 & $184.5 \%$ & $194.2 \%$ & $174.2 \%$ & $166.1 \%$ \\
\hline
\end{tabular}




\section{Results}

\section{Patient and lesion characteristics}

This study included 23 eligible NMSCs in 19 patients. The mean age was 68 years. Seventeen patients were $\geq 60$ years old. All patients were Caucasian. Twenty lesions were BCC and 3 lesions were SCC. All tumors were staged as $\mathrm{T} 1$ or $\mathrm{T} 2$. The most common location was the nose (10 lesions). The characteristics of 23 lesions are summarized in Table 2.

\section{Measurement of lateral extension and depth of non-melanomatous skin cancer using ultrasound images}

Normal anatomy: The layers of skin: epidermis (thickness 0.06-0.6 mm), dermis (thickness 1-4 mm), and hypodermis (subcutaneous tissues; thickness 5-20 mm) can be visualized on US. The epidermis appears as the most superficial, well-defined, hyperechoic, linear band producing the "entry echo" between the US gel and skin (epidermal entry echo [EEE]). The dermis below the epidermis is also hyperechoic, usually less echogenic than epidermis, and with hypoechoic hair follicles, vessels, and sebaceous glands. The hypodermis is hypoechoic with intervening hyperechoic connective tissue septa separating fat lobules. Underneath the skin, superficial fascia covering muscle may be identified as a linear hyperechoic structure $[29,30]$. Figure $2 B$ illustrates each layer described on US.

\section{Ultrasound of non-melanomatous skin cancer}

US provides $2 \mathrm{D}$ or $3 \mathrm{D}$ images of deep dermal or subdermal layers by measuring differences in sound impedance [31]. On high resolution US, BCC is a solid, heterogeneous and hypoechoic mass that shows irregular margins containing echogenic foci of keratin nests. SCC also appears hypoechoic but may be more aggressive at the time of presentation with infiltration of deeper tissues $[29,30]$. Figure $2 A$ and B shows a NMSC on the forehead and US image depicting the clarity and capability of US in providing measurements for eBT prescriptions.

\section{D measurements with ultrasound and electronic brachytherapy}

The mean largest diameter of all lesions was $8 \mathrm{~mm}$ (range 2.6-20 mm). The mean depth of all lesions was $2.1 \mathrm{~mm}$ (range 1-3.4 mm). For BCC, the largest lateral extent ranged from 2.6-12 $\mathrm{mm}$ and depth ranged from 1-3.4 $\mathrm{mm}$. For SCC, the largest lateral extent ranged from $8-20 \mathrm{~mm}$ and depth ranged from $2.5-3.1 \mathrm{~mm}$. The only upper extremity lesion had the largest lateral extent $(20 \mathrm{~mm})$. With US depth measurements, prescription depth could be rounded or prescribed to the fractional depth (e.g. $1.5 \mathrm{~mm}$ rather than every millimeter). Figure $3 \mathrm{~A}$ and $\mathrm{B}$ shows the largest lateral extent and the second largest lateral extent, and also depicts the largest diameter and depth for each lesion using US-determined measurements for eBT. No correlation between location, lateral size, and depth was seen in our study.

\section{Results of treatment}

All patients have had a complete response, and with a median follow-up of 12 months (range 6-22 months), there have been no failures. More importantly, there have been no cases of grade 4 skin necrosis or delayed healing. Figure 4 shows progressively worsening radiation-induced erythema during eBT for a patient with BCC. This grade 2 erythema usually resolves by the 1 month follow-up.

\section{Discussion}

In this methodology paper, we describe the use of commonly available US for determining the lateral extent and depth of a pathologically proven NMSC, and the clinical application of such measurements to guide eBT planning. Without a standard objective mode of measuring NMSCs, many clinicians determine prescription depth and applicator size based on clinical judgment $[6,7]$ or biopsy information [26]. When using small contact appli-

Table 2. Characteristics of non-melanomatous skin cancer lesions

\begin{tabular}{|c|c|c|}
\hline & \# Lesions & $\%$ Lesions \\
\hline \multicolumn{3}{|l|}{ Histology } \\
\hline $\mathrm{BCC}$ & 20 & 87 \\
\hline SCC & 3 & 13 \\
\hline \multicolumn{3}{|l|}{ Tumor stage } \\
\hline $\mathrm{T} 1$ & 12 & 52.2 \\
\hline $\mathrm{T} 2$ & 11 & 47.8 \\
\hline \multicolumn{3}{|c|}{ Location of lesion } \\
\hline Nose & 10 & 43.5 \\
\hline Cheek & 3 & 13 \\
\hline Forehead & 2 & 8.7 \\
\hline Scalp & 2 & 8.7 \\
\hline Lip & 2 & 8.7 \\
\hline Extremity & 2 & 8.7 \\
\hline Ear & 1 & 4.3 \\
\hline Abdomen & 1 & 4.3 \\
\hline \multicolumn{3}{|l|}{ Depth (mm) } \\
\hline $1-1.9$ & 9 & 39.1 \\
\hline $2-2.9$ & 8 & 34.7 \\
\hline $3-3.9$ & 6 & 26 \\
\hline 4-4.9 & 0 & 0 \\
\hline$\geq 5$ & 0 & 0 \\
\hline \multicolumn{3}{|c|}{$\begin{array}{l}\text { Largest lateral } \\
\text { Extent }(\mathrm{mm})\end{array}$} \\
\hline $1-5$ & 4 & 17.3 \\
\hline $6-10$ & 16 & 69.5 \\
\hline$\underline{11-15}$ & 1 & 4.3 \\
\hline $16-20$ & 2 & 8.7 \\
\hline
\end{tabular}

$B C C$ - basal cell carcinoma, SCC - squamous cell carcinoma 

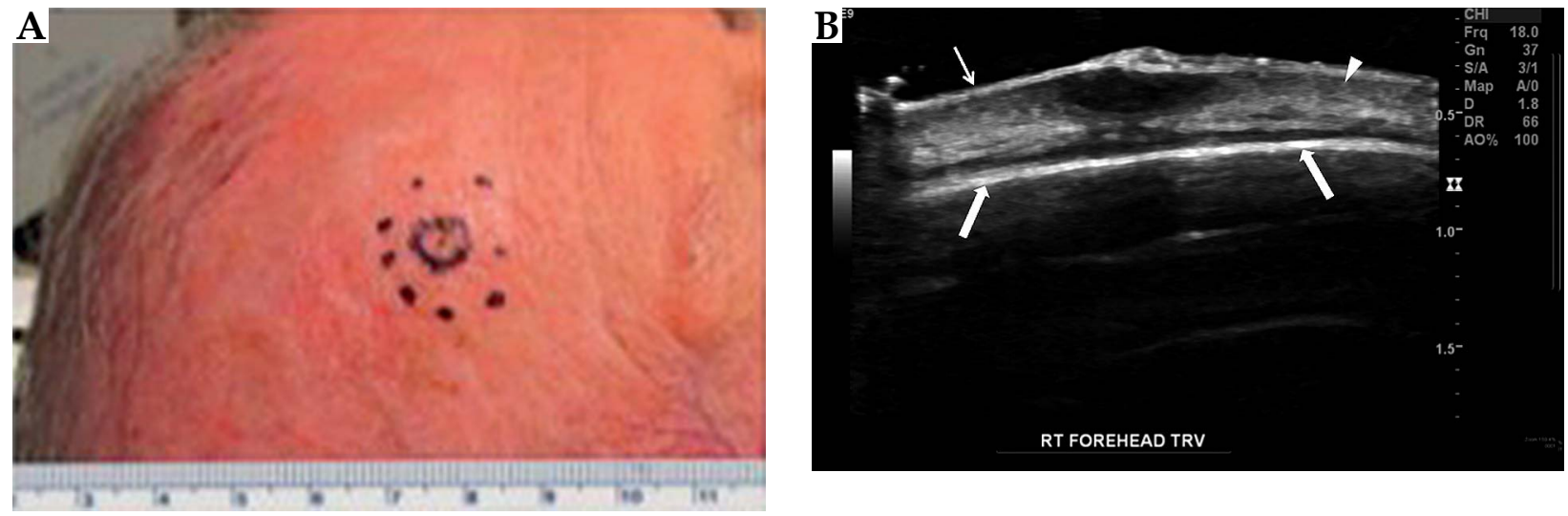

Fig. 2. Clinical setup and ultrasound image. A right forehead basal cell carcinoma (A) delineated with a pen (dotted line) and (B) on ultrasound found to have a hypoechoic lesion that measures $7.5 \mathrm{~mm}$ in the transverse dimension, $3 \mathrm{~mm}$ in depth and $5 \mathrm{~mm}$ in the sagittal dimension (not shown). A $20 \mathrm{~mm}$ applicator was chosen to treat to a depth of $3 \mathrm{~mm}$. A hyperechoic epidermis (thin arrow), a hypoechoic dermal layer with a more echoic signal from subcutaneous fat beneath (arrowhead), and the strongly echoic layer below subcutaneous fat is bone that creates shadowing so nothing deeper is anatomically distinguishable (thick arrows)

A

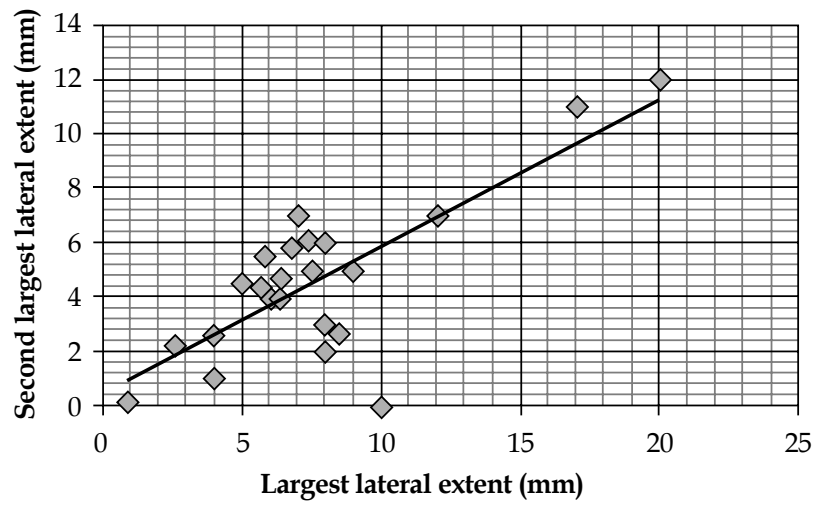

B

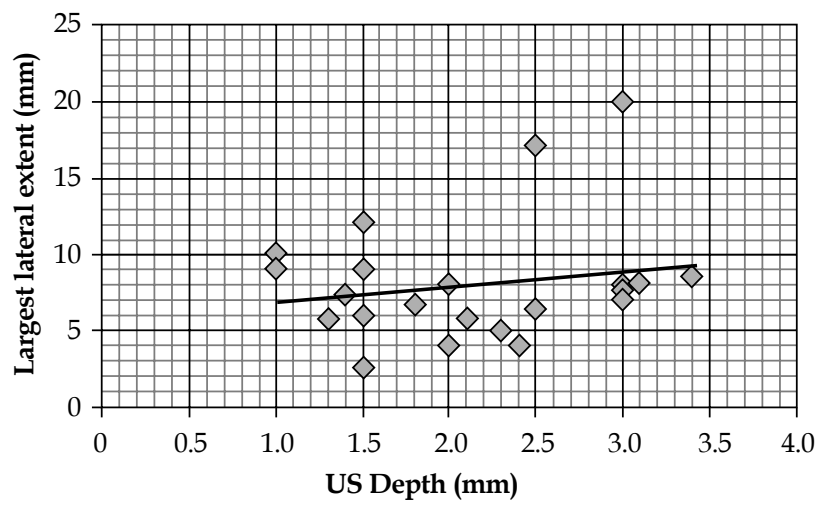

Fig. 3. Ultrasound (US) measurements of lateral extensions and depth. The largest lateral extent ( $\mathrm{mm}$ ) and second largest lateral extent $(\mathrm{mm})$ of each non-melanomatous skin cancer measured with US and used for electronic brachytherapy planning are shown (A). The largest lateral extension $(\mathrm{mm})$ and depth $(\mathrm{mm})$ of each non-melanomatous skin cancer measured with US and used for electronic brachytherapy planning are shown (B)
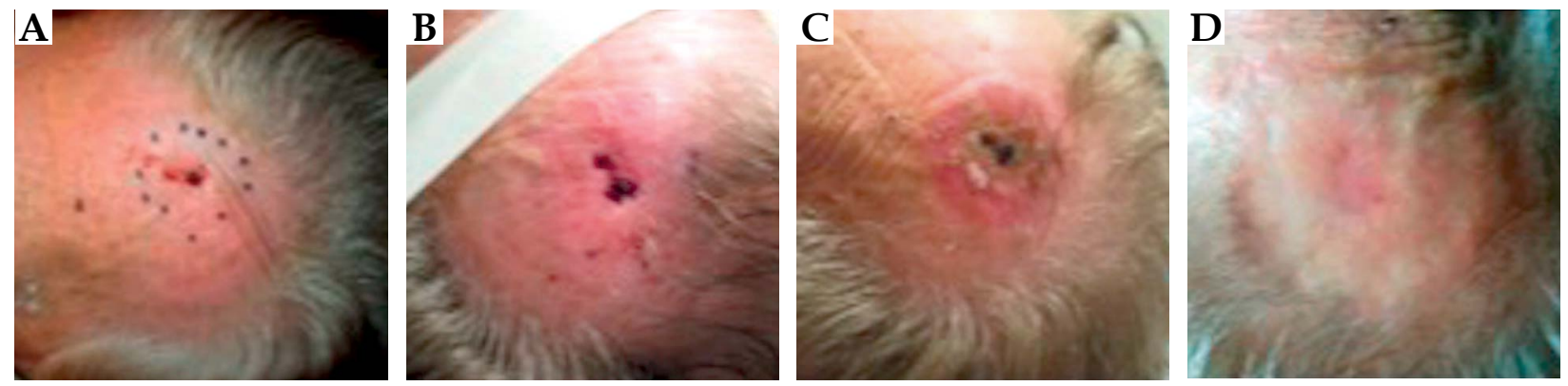

Fig. 4. Non-melanomatous skin cancer treated with electronic brachytherapy. A scalp lesion measured to be $6.3 \mathrm{~mm} \times 4 \mathrm{~mm} \times$ $2 \mathrm{~mm}$ with ultrasound was treated with a $35 \mathrm{~mm}$ applicator to a $2 \mathrm{~mm}$ depth. The dose regimen was $40 \mathrm{~Gy}$ in 10 fractions every other day and the lesion is shown: (A) prior to initial treatment, (B) after 5 fractions, (C) after completion of 10 fractions, and (D) after 1 month follow-up. There was progressively worsening erythema over the course of electronic brachytherapy that resolved after 1 month

cators, this clinical approach bears potential issues such as inadequate lateral coverage and/or under-treating the NMSC or the alternative of over-treatment, which may lead to unnecessary toxicity (skin necrosis or delayed healing). Ballester-Sánchez et al. [26] used punch biopsy then US in order to evaluate depth for NMSC. However, 
this leads to questions about whether the punch biopsy removes the deepest portion of the lesion prior to US measurement for eBT. In our study, shave biopsies were used for pathologic verification allowing a greater chance that the remaining NMSC depth and margins were adequately assessable by US.

Unfortunately, there are few available modalities that can be used for non-invasive and accurate measurement of a superficial lesion's lateral extent and depth. A prior study has discussed US use for NMSC treated with HDR-BT [13]. There has been report of using CT for depth assessment in prior eBT studies $[17,18]$ but in our experience, CT does not have enough discrimination for accurate depth determination when dealing with superficial thin lesions.

We conducted this pilot study to provide an element of QA and guidelines for accurate treatment planning that is currently not performed routinely rather than proving clinical outcomes. We have found that US evaluation can visualize and measure thin NMSCs in the range of 1-5 $\mathrm{mm}$. Thus, we advocate that rather than using clinical exam measurements (the current standard of care), which can be subjective based on practitioner, objective depth, and lateral extent US measurements that should be used as guidance when prescribing radiotherapy for these superficial cancers. The detailed use of US for NMSC eBT planning has not been described before and is an important consideration for future eBT skin cancer guidelines.

Our intent for this conceptual paper is to describe a more standard technique for prescribing eBT for NMSC; however, we do feel it is important to provide our preliminary clinical outcomes data in order to show that the use of US does not worsen local control or cosmesis compared to previously published studies $[17,18]$. Compared to previously published data with median follow-ups ranging from 4.1 to 10 months, the 1 year patient outcomes data for our study shows $100 \%$ local control and no significant toxicity, essentially similar to reported data by other studies $[17,18,22,26]$. Only long-term follow-up will determine whether US-guided prescriptions will result in better cosmetic outcomes due to the precision of depth prescriptions. A novel point from our study is that with the use of US, physicians are not limited to depth prescriptions of every millimeter (i.e. $3 \mathrm{~mm}$ or $4 \mathrm{~mm}$ ) but can prescribe eBT to tenths of millimeters (i.e. $2.7 \mathrm{~mm}$ or $3.5 \mathrm{~mm}$ ) in order to spare superficial layers from receiving higher doses with unnecessary deeper prescriptions.

A limitation of current commercially available US is its capacity to detect NMSCs that are $<1 \mathrm{~mm}$ deep. Five biopsy-proven NMSCs could not be detected by our US modality. A high frequency ultrasound unit (HFUS) such as $\geq 50 \mathrm{MHz}$ may have better resolution to detect previously undetectable lesions, and improve the quality and detail of these radiologic images [30,32]. HFUS could also be a useful tool for defining diameter and characterizing changes over time. Various other clinical imaging modalities for evaluating NMSC have been described such as dermoscopy, optical coherence tomography, and reflectance confocal microscopy [33, 34, 35, 36, 37, 38, 39] but each has its own caveats.

Standard imaging with a commonly available US unit eliminates guesswork at the time of prescribing and planning eBT. There are no studies to our knowledge that have reported on the systematic use of US measurements of depth and lateral extension for eBT treatment planning. This study may bode importance in further clinical applications for adequately treating and following NMSC patients. We feel that as the use of eBT in the treatment of NMSC continues to grow, it becomes more important to have standard measurement techniques for prescribing treatment and reporting outcome data.

\section{Conclusions}

Ultrasound technology provides an easy and objective modality to determine the diameter and depth of a lesion when treating thin NMSC lesions with Xoft eBT. The suggested guidelines provide a set of simple parameters to follow when treating superficial skin cancers over cosmetically sensitive parts of the body where skin toxicity could jeopardize the anticipated good cosmetic results.

\section{Disclosure}

Authors report no conflict of interest.

\section{References}

1. Rogers HW, Weinstock MA, Harris AR et al. Incidence estimate of nonmelanoma skin cancer in the United States, 2006. Arch Dermatol 2010; 146: 283-287.

2. What you need to know about melanoma and other skin cancers. NIH Publication No 10-7625; available at: http:/ / www.cancer.gov/publications/patient-education/skin.pdf. Accessed October 21, 2014.

3. Guix B, Finestres F, Tello JI et al. Treatment of skin carcinomas of the face by high-dose-rate brachytherapy and custom-made surface molds. Int J Radiat Oncol Biol Phys 2000; 47: 95-102.

4. Locke J, Karimpour S, Young G et al. Radiotherapy for epithelial skin cancer. Int J Radiat Oncol Biol Phys 2001; 51: 748-755.

5. Sedda A, Rossi G, Cipriani C et al. Dermatological high-doserate brachytherapy for the treatment of basal and squamous cell carcinoma. Clin Exp Dermatol 2008; 33: 745-749.

6. Lovett RD, Perez CA, Shapiro SJ et al. External radiation of epithelial skin cancer. Int J Radiat Oncol Biol Phys 1990; 19: 235-242.

7. Kwan W, Wilson D, Moravan V. Radiotherapy for locally advanced basal cell and squamous cell carcinomas of the skin. Int J Radiat Oncol Biol Phys 2004; 60: 406-411.

8. Malinverni G, Stasi M, Baiotto B et al. Clinical application and dosimetric calibration procedure of the superficial and orthovoltage therapy unit Therapax DXT300. Tumori 2002; 88: 331-337.

9. Rong Y, Zuo L, Shang L, Bazan JG. Radiotherapy treatment for nonmelanoma skin cancer. Expert Rev Anticancer Ther 2015; 15: 765-776.

10. Svoboda V, Kovarik J, Morris F. High dose-rate microselectron molds in the treatment of skin tumors. Int J Radiat Oncol Biol Phys 1995; 31: 967-972.

11. Rio E, Bardet E, Ferron $C$ et al. Interstitial brachytherapy of periorificial skin carcinomas of the face: a retrospective study of 97 cases. Int J Radiat Oncol Biol Phys 2005; 63: 753-757.

12. Conill C, Sánchez-Reyes A, Molla M et al. Brachytherapy with 192Ir as treatment of carcinoma of the tarsal structure of the eyelid. Int J Radiat Oncol Biol Phys 2004; 59: 1326-1329.

13. Tormo A, Celada F, Rodriguez $S$ et al. Non-melanoma skin cancer treated with HDR Valencia applicator: clinical outcomes. J Contemp Brachytherapy 2014; 6: 167-172. 
14. Pérez-Calatayud J, Granero D, Ballester F et al. A dosimetric study of Leipzig applicators. Int J Radiat Oncol Biol Phys 2006; 62: 579-584.

15. Niu H, Hsi WC, Chu JC et al. Dosimetric characteristics of the Leipzig surface applicators used in the high dose rate brachy radiotherapy. Med Phys 2004; 31: 3372-3377.

16. Treating with eBx. Xoft Inc.; available at: http://www.xoftinc.com/treating_derma.html. Accessed October 24, 2014.

17. Bhatnagar A, Loper A. The initial experience of electronic brachytherapy for the treatment of non-melanoma skin cancer. Radiat Oncol 2010; 5: 87.

18. Bhatnagar A. Nonmelanoma skin cancer treated with electronic brachytherapy: results at 1 year. Brachytherapy 2013; 12: 134-140.

19. Technical white paper: $\mathrm{H}-36$ task force on electronic brachytherapy. Conference of Radiation Control Program Directors, Inc. Available at: http:/ / www.crcpd.org/Pubs/H-36BrachytherapyWhitePaper.pdf. Accessed on June 15, 2015.

20. Arterbery VE, Watson AC. An electronic brachytherapy technique for treating squamous cell carcinoma in situ of the digit: a case report. BMC Research Notes 2013; 6: 147.

21. Pons-Llanas O, Ballester-Sánchez R, Celada-Álvarez FJ et al. Clinical implementation of a new electronic brachytherapy system for skin brachytherapy. J Contemp Brachytherapy 2015; 6: 417-423.

22. Ballester-Sánchez R, Pons-Llanas O, Candela-Juan C et al. Efficacy and safety of electronic brachytherapy for superficial and nodular basal cell carcinoma. J Contemp Brachytherapy 2015; 7: 231-238.

23. Garcia-Martinez T, Chan JP, Perez-Calatuyad J et al. Dosimetric characteristics of a new unit for electronic skin brachytherapy. J Contemp Brachytherapy 2014; 6: 45-53.

24. Rong Y, Welsh JS. Surface applicator calibration and commissioning of an electronic brachytherapy system for nonmelanoma skin cancer treatment. Med Phys 2010; 37: 5509-5517.

25. Ma CM, Coffey CW, DeWerd LA et al. TG-61 AAPM protocol for 40-300 kV x-ray beam dosimetry in radiotherapy and radiobiology. Med Phys 2001; 28: 868-893.

26. Ballester-Sánchez R, Pons-Llanas O, Llavador-Ros $M$ et al. Depth determination of skin cancers treated with superficial brachytherapy: ultrasound vs. histopathology. J Contemp Brachytherapy 2015; 6: 356-361.

27. Compton CC, Byrd DR, Garcia-Aguilar J et al. (eds.). AJCC Cancer Staging Atlas. A Companion to the Seventh Editions of the AJCC Cancer Staging Manual and Handbook. Springer, New York 2012.

28. Pasquali P, Camacho E, Fortuny A. Use of $22 \mathrm{MHz}$ High-Frequency Ultrasound in the Management of Skin Cancer. In: Skin Cancer, Current Clinical Pathology. Baldi A et al. (eds.). Springer Science + Business Media New York, New York 2014; pp. 245-256.

29. Wortsman X. Common applications of dermatologic sonography. J Ultrasound Med 2012; 31: 97-111.

30. Mandava A, Ravuri PR, Konathan R. High-resolution ultrasound imaging of cutaneous lesions. Indian J Radiol Imaging 2013; 23: 269-277.

31. Rohrbach DJ, Muffoletto D, Huihui J et al. Preoperative mapping of nonmelanoma skin cancer using spatial frequency domain and ultrasound imaging. Acad Radiol 2014; 21: 263-270.

32. Guittet C, Ossant F, Remenieras JP et al. High-frequency estimation of the ultrasonic attenuation coefficient slope obtained in human skin: Simulation and in vivo result. Ultrasound Med Biol 1999; 25: 421-429.

33. Binder M, Puespoeck-Schwarz M, Steiner A et al. Epiluminescence microscopy of small pigmented skin lesions: shortterm formal training improves the diagnostic performance of dermatologists. J Am Acad Dermatol 1997; 36 (2 Pt 1): 197-202.
34. Grin CM, Kopf AW, Welkovich B et al. Accuracy in the clinical diagnosis of malignant melanoma. Arch Dermatol 1990; 126: 763-766.

35. Mayer J. Systematic review of the diagnostic accuracy of dermatoscopy in detecting malignant melanoma. Med J Aust 1997; 167: 206-210.

36. Miller M, Ackerman AB. How accurate are dermatologists in the diagnosis of melanoma? Degree of accuracy and implications. Arch Dermatol 1992; 128: 559-560.

37. Wolf IH, Smolle J, Soyer HP et al. Sensitivity in the clinical diagnosis of malignant melanoma. Melanoma Res 1998; 8: 425429.

38. Gambichler T, Regeniter P, Bechara FG et al. Characterization of benign and malignant melanocytic skin lesions using optical coherence tomography in vivo. J Am Acad Dermatol 2007; 57: 629-637.

39. O'Donnell AT, Kim CC. Update and clinical use of imaging technologies for pigmented lesions of the skin. Semin Cutan Med Surg 2012; 31: 38-44. 